\title{
Association Between Nicotine Dependence Severity, BOLD Response to Smoking Cues, and Functional Connectivity
}

\author{
Eric D Claus*,', Sara K Blaine ${ }^{2}$, Francesca M Filbey ${ }^{3}$, Andrew R Mayer' and Kent E Hutchison ${ }^{1,2}$ \\ 'The Mind Research Network, Albuquerque, NM, USA; ${ }^{2}$ University of Colorado at Boulder, Boulder, CO, USA; ${ }^{3}$ Center for BrainHealth, University \\ of Texas at Dallas, Dallas, TX, USA
}

\begin{abstract}
Enhanced motivational salience towards smoking cues is a consequence of chronic nicotine use, but the degree to which this value increases beyond that of other appetitive cues is unknown. In addition, it is unclear how connectivity between brain regions influences cue reactivity and how cue reactivity and functional connectivity are related to nicotine dependence severity. This study examined neural responses during the presentation of smoking cues and appetitive control cues, as well as functional connectivity in II 6 smokers with a range of nicotine dependence severity. Smoking cues elicited greater response above baseline than food cues in orbitofrontal cortex (OFC) and supplementary motor area (SMA) and less deactivation below baseline in middle frontal gyrus, inferior parietal lobe, and middle temporal gyrus. Psychophysiological interaction (PPI) analysis using right OFC as a seed revealed increased connectivity with somatosensory cortex and lateral inferior parietal lobe during smoking cues compared with food cues. Similarly, a PPI analysis using left insula as a seed showed stronger connectivity with somatosensory cortex, right insula, OFC, and striatum. Finally, relationships with nicotine dependence scores showed enhanced response in insula and dorsal anterior cingulate cortex in the smoking vs food comparison, and increased connectivity between insula and circuits involved in motivated behavior. Combined, these results suggest that smokers engage attentional networks and default mode networks involved in self-referential processing to a greater degree during smoking cues. In addition, individuals with greater nicotine dependence severity show increased engagement of sensorimotor and motor preparation circuits, suggesting increased reliance on habitual behavior.

Neuropsychopharmacology (2013) 38, 2363-2372; doi:I0.1038/npp.2013.134; published online 19 June 2013
\end{abstract}

Keywords: craving; insula; functional connectivity; nicotine dependence

\section{INTRODUCTION}

Several studies have examined neural responses to cigaretterelated cues in an effort to develop biologically-based measures of cue reactivity, and focused on regions such as the dorsolateral prefrontal cortex (DLPFC), anterior cingulate cortex (ACC), striatum, and orbitofrontal cortex (OFC) (Wilson et al, 2005; Janes et al, 2009; Brody et al, 2002; Franklin et al, 2007). These regions are implicated in cognitive control as well as assigning motivation value to stimuli, making them important components of craving networks. In addition, a meta-analysis highlighted the precuneus and early visual processing regions as important contributors to cue reactivity in smokers, through their role in attentional bias towards smoking cues (Engelmann et al, 2012). Other studies also suggest that individuals with lesions to the insula exhibited much higher rates of successful smoking cessation relative to individuals with lesions in other regions (Naqvi et al, 2007). The insula has

\footnotetext{
*Correspondence: Dr ED Claus, The Mind Research Network, II0 I Yale Boulevard NE, Albuquerque, NM 87106, USA, Tel: + I 505 272 5028, Fax: + I 505272 8002, E-mail: eclaus@mm.org Received II January 20 I3; revised 9 May 20 I3; accepted I 0 May 20 I3; accepted article preview online 24 May 2013
}

been hypothesized to have a significant role in interoception awareness, and thus may have a casual role in maintaining addiction to nicotine through its representation of negative bodily experiences associated with withdrawal and craving (Naqvi and Bechara, 2009).

While previous studies have controlled for the visual aspects of smoking cues, few, if any, studies have compared neural responses during cigarette cues to motivationally significant appetitive cues. During comparisons of motivationally salient cues such as cigarette-related pictures to a neutral object such as a pencil (Wilson et al, 2005), it is difficult to know to what extent the cigarette cue produces a greater response solely because it is an appetitive cue. Therefore, the degree to which cigarette cues have gained a competitive edge over primary appetitive reinforcers is largely unknown. However, it is well known that appetitive cues strongly elicit blood oxygen level-dependent (BOLD) response in many of the regions typically observed in studies of drug cue reactivity such as OFC, insula, striatum, and amygdala (Tang et al, 2012). Further, a recent study suggested that severe nicotine dependence is associated with reduced response to secondary reinforcers (ie, money), rather than enhanced response to cigarette-related cues (Bühler et al, 2010). In addition, adolescent smokers show reduced neural responses to pleasurable foods compared 
with non-smoking controls, suggesting reward deficiency (Rubinstein et al, 2011). Both studies therefore suggest that motivational value for other rewards is dampened, but it may be possible that the difference between responses to cigarette cues and food cues is increased as a function of nicotine dependence severity. For example, dependence severity is associated with greater neural response to smoking compared with neutral cues in motor planning circuits (dorsal ACC, caudate, globus pallidus, right middle frontal gyrus/premotor cortex), suggesting that more severely dependent individuals engage enhanced approach motivation when viewing motivationally salient stimuli compared to content with no motivational value (Smolka et al, 2006; McClernon et al, 2008).

In addition, few studies to date have examined how functionally connected networks of brain regions engaged during task performance contribute to craving responses. While studies of intrinsic functional connectivity in nicotine users have shown evidence of decreased connectivity in circuits involving the dorsal striatum and dorsal ACC at rest (Hong et al, 2010), few studies have investigated task-based functional connectivity in smokers. In one study of smokers seeking cessation treatment, a functionally connected network that included bilateral insula, dorsal ACC, and striatum showed enhanced response in individuals who remained abstinent than those who relapsed, which may have resulted from reduced ability to regulate emotion in relapsers (Janes et al, 2010). The degree to which functionally connected networks that include insula and dACC nodes differentially react to smoking cues as opposed to other motivationally significant cues is not clear and there is a lack of knowledge regarding the influence of nicotine dependence severity on functionally connected networks during cue presentation.

This study was designed to test the degree to which smoking cues differentially engage reward circuits compared with appetitive control cues and how this engagement may vary among smokers with varying levels of nicotine dependence. In addition, we examined the networks of brain regions that are functionally connected and respond preferentially to smoking cues, and determined how dependence severity moderates these networks. We hypothesized that smoking cues would elicit greater neural responses in the ACC, OFC, insula, dorsal and ventral striatum than normal appetitive cues and that increased dependence would be associated with an increased difference in these regions. In addition, we hypothesized that functional connectivity during smoking cues as compared with food cues between insula and AACC would be stronger in individuals who were more severely nicotine dependent.

\section{MATERIALS AND METHODS}

\section{Participants}

One hundred and thirty-two right-handed individuals (52 females) between the ages of 18 and 55 (mean age $=31.36$ years) who reported smoking cigarettes in the past 90 days participated in the study. This sample was chosen to include a wide range of dependence levels to better understand the relationship between cue-related activity and dependence severity. Potential participants were excluded if they reported receiving treatment for or diagnosis of a psychiatric illness, prior head injury, use of medications that impact the central nervous system, or any contraindications for participating in an MRI study (eg, pregnancy, nonremovable metallic implants).

\section{Procedures}

Institutional Review Board-approved informed consent was obtained for all participants. Quantity and frequency information regarding tobacco, alcohol, and marijuana use were acquired with the Time Line Follow Back procedure (Sobell and Sobell, 1992). Nicotine dependence severity was assessed using the Fagerstrom test for nicotine dependence (FTND) (Heatherton et al, 1991). Handedness was assessed using the Edinburgh handedness questionnaire (Oldfield, 1971).

Participants abstained from smoking for $2 \mathrm{~h}$ before the scanning session; all participants arrived $1 \mathrm{~h}$ before scanning commenced, bringing the total amount of abstinence to approximately $3 \mathrm{~h}$. This abstinence period was chosen to minimize the effects of acute nicotine exposure on BOLD responses while minimizing severe withdrawal effects that could occur with extended periods of abstinence. Before the scan commenced, participants completed the Wisconsin Smoking Withdrawal Scale (Welsch et al, 1999).

\section{Cue-Exposure Task}

The cue-exposure task consisted of two 6-min runs with pseudorandom presentations of seven different smokingrelated videos and seven different food-related videos. Thus, for the entire experiment, there were a total of 14 smoking trials and 14 food trials. Each video ranged in length from 7 to $14 \mathrm{~s}$; the average time of smoking and food videos was not different. Both the smoking and food-related videos were chosen to represent preparation of the stimulus for subsequent ingestion and also the act of ingestion itself. Between the videos, participants viewed a fixation cross for variable times to introduce jitter into the design and improve the fit of the general linear model (Jezzard et al, 2001). Stimulus presentation was controlled using Presentation (www.neurobs.com), and stimuli were presented to participants using a rear projection mirror system.

\section{MRI Acquisition}

All scans were acquired on a Siemens 3T Trio scanner located at the Mind Research Network. An echo-planar gradient-echo pulse sequence $(\mathrm{TR}=2000 \mathrm{~ms}$; $\mathrm{TE}=29$; flip angle $=75^{\circ}$ ) was acquired with an 8-channel head coil, and images were acquired parallel to the ventral surface of a participant's OFC to reduce signal dropout and distortion in this region (Deichmann et al, 2003). Each volume acquired consisted of 33 axial slices $(64 \times 64$ matrix, $3.75 \times 3.75 \mathrm{~mm} 2$, $3.5 \mathrm{~mm}$ thickness, $1.05 \mathrm{~mm}$ gap). In addition, a highresolution multiecho T1-weighted MP-RAGE anatomical image was acquired $(\mathrm{TR}=2530 \mathrm{~ms} ; \mathrm{TE}=1.64,3.5,5.36$, 7.22 , and $9.08 \mathrm{~ms}$; flip angle $=7^{\circ} ; 192$ sagittal slices; $256 \times 256$ matrix; slice thickness $=1 \mathrm{~mm}$, no gap) for each participant. 


\section{fMRI Analysis}

The first three volumes of each functional run were discarded to remove saturation effects. MCFLIRT (FMRIB) (Jenkinson et al, 2002) was used to motion correct images; motion parameters were used in subsequent analyses. Sixteen participants were dropped from the analysis for motion that exceeded $2 \mathrm{~mm}$ /degrees, for a final sample size of 116 participants. The time series was deskulled using BET (Smith, 2002), spatially smoothed with a 5-mm full-width half-max Gaussian kernel, temporally filtered using a highpass filter of $100 \mathrm{~s}$, and grand mean intensity normalized across the whole brain with a single value; all steps were performed using FMRIB's Expert Analysis Tool (Smith et al, 2004).

Customized square waveforms representing the condition of interest (food $v s$ smoking) and the duration of stimulus presentation were convolved with a double gamma hemodynamic response function. Time-series analyses were conducted using FMRIB's Improved Linear Model (FILM) with local autocorrelation estimation (Woolrich et al, 2004, 2001). This first-level analysis generated parameter estimates for each condition of interest, Food and Smoking. Using the parameter estimates for each condition, we computed contrasts of Smoking $>$ Baseline, Food $>$ Baseline, Smoking $>$ Food, and Food $>$ Smoking for each participant. Each contrast map was registered to a common template (MNI152 brain) using a two-step registration process in FMRIB's Linear Image Registration Tool (FLIRT) (Jenkinson et al, 2002). First-level analyses for each run were combined at the subject level using a fixed-effect model.

\section{Functional Connectivity}

To investigate functional connectivity during cue reactivity, we used a psychophysiological interaction (PPI) (Friston et al, 1997; O'Reilly et al, 2012) approach focused on an ROI in right OFC (rOFC), a region that showed significant effects in the main-effect analysis comparing Smoking and Food videos, and also left insula, a region that showed a significant correlation with FTND scores in the Smoking > Food contrast. For each ROI, spheres with a $6 \mathrm{~mm}$ radius were drawn around peaks of activation from the main-effect analysis (rOFC: $x=46, y=34, z=-6$; left insula: $x=-40$, $\mathrm{y}=12, z=6$ ) that minimized the inclusion of adjacent white matter. To extract the time series, the standard space ROI was first transformed into scanner space using transformation matrices from the registration described above. The ROI time series was extracted from the motion corrected and temporally filtered time series. The corresponding time series was entered into the following model that included motion covariates:

Signal $=($ Smoking - Food $)+$ ROI $+($ Smoking - Food $) \times$ $\mathrm{ROI}+($ Smoking + Food $)+x+y+z+$ pitch + roll + yaw

For each run of the task, the PPI model was estimated using FILM (Woolrich et al, 2004), which produced betas for each regressor. Group analyses focused on the interaction term (Smoking - Food $\times$ ROI), which represents those regions that show a stronger correlation with the seed ROI during smoking videos compared with food videos.

\section{Group Analysis}

Group analyses were conducted using FMRIBs Local Analysis of Mixed Effects (FLAME) Stage 1. We examined all main effects (ie, Smoking - Food, Smoking - Food $\times$ rOFC, Smoking - Food $\times$ left Insula) using a one-sample $t$-test. In addition, first-order correlations were conducted to examine the influence of nicotine dependence severity on the contrasts above. Each of the analyses was also controlling for withdrawal as measured by the WSWS (see Supplementary Figures 1 and 3 and Supplementary Tables 1 and 3). Finally, multiple regressions were used to examine the influence of nicotine dependence severity on BOLD response while controlling for marijuana use, alcohol use (drinks per drinking day), and age (see Supplementary Figures 2 and 4 and Supplementary Tables 2, 4, and 5). To correct for multiple comparisons, group-level results were thresholded at $p<0.01$ and minimum cluster size of 239 voxels for a corrected level of $p<0.05$ as determined by Monte Carlo simulations (3dClustSim in $\mathrm{AFNI}$ ).

\section{RESULTS}

\section{Cigarette and Drug Use}

Participants were moderately nicotine dependent as evidenced by FTND scores $($ mean $=3.9(2.7)$ ) and average cigarettes smoked per day $($ mean $=14(7.3))$. The wide range of FTND scores (0-9) and cigarettes smoked per day (1-31) make this an ideal sample for identifying how variation in dependence severity contributes to neural responses during cue-elicited craving. Subjects consumed an average of 5.1 (3.9) drinks per drinking day and $46 \%$ smoked marijuana at least one time per week in the past month.

\section{fMRI: Main Effects}

In the primary contrast of Smoking vs Food, we found greater neural response in bilateral OFC, medial frontal gyrus/SMA, middle frontal gyrus, inferior parietal lobe, and middle temporal gyrus during smoking videos compared with food videos $(z>3.09$, cluster corrected $p<0.05$; see Table 1 and Figure 1a). Only the bilateral OFC, bilateral IPL, and SMA showed significant increases above baseline during the smoking videos. In all other cases, responses during smoking videos resulted in less deactivation below baseline compared with the food videos.

In contrast, Food videos elicited greater response than Smoking videos in several regions including the entire bilateral visual cortex, inferior parietal cortex, pre/postcentral gyrus, middle frontal gyrus, frontal pole, and striatum $(z>3.09$, cluster corrected $p<0.05$; Table 1 and Figure 1a). Examination of the beta maps for Food (and Smoking) videos $v s$ baseline suggested that all differences were the result of the Food videos eliciting responses above baseline to a greater degree than that observed in the Smoking videos. In no case was the significant difference the result of reduced deactivation below baseline (Figure 1b, c). 
Table I Regions Showing Greater Response During Smoking Videos Than Food Videos, Food Greater Than Smoking, and Positive Relationships Between FTND Scores and the Smoking > Food Contrast

\begin{tabular}{|c|c|c|c|c|c|c|c|}
\hline Contrast & Region & BA & $\operatorname{Max} Z$ & Voxels & $x$ & $y$ & z \\
\hline \multirow[t]{8}{*}{ Smoking > Food } & Medial frontal gyrus (L) & 10 & 6.61 & 7670 & -10 & 48 & 6 \\
\hline & Supramarginal gyrus, posterior division $(L)$ & 40 & 10 & 6839 & -60 & -46 & 32 \\
\hline & Posterior cingulate gyrus $(L)$ & 23 & 7.05 & 3400 & 0 & -20 & 34 \\
\hline & Supramarginal gyrus, posterior division (R) & 40 & 8.75 & 2684 & 58 & -44 & 30 \\
\hline & Left crus ॥ (L) & - & 6.28 & 779 & -20 & -80 & -36 \\
\hline & Right crus II (R) & - & 6.09 & 497 & 22 & -86 & -36 \\
\hline & Middle frontal gyrus (R) & 6 & 4.83 & 277 & 42 & 10 & 56 \\
\hline & Orbitofrontal cortex (R) & 47 & 4.04 & 112 & 32 & 24 & -22 \\
\hline & Frontal pole $(\mathrm{R})$ & 46 & 6.01 & 420 & 46 & 44 & 12 \\
\hline & Orbitofrontal cortex $(\mathrm{L})$ & 47 & 5.39 & 240 & -28 & 32 & -16 \\
\hline \multirow[t]{4}{*}{ FTND correlation } & Middle temporal gyrus, temporo-occipital part $(L)$ & 21 & 4.15 & 675 & -44 & -46 & 6 \\
\hline & Insula/temporal pole $(L)$ & 21 & 3.68 & 365 & -56 & 10 & -12 \\
\hline & Planum temporale $(\mathrm{L})$ & 41 & 3.96 & 317 & -58 & -20 & 8 \\
\hline & Dorsal ACC $(L)$ & 6 & 3.51 & 309 & -18 & 12 & 52 \\
\hline
\end{tabular}

Coordinates are presented in MNI space. BA_Brodmann area; max Z-peak $z$-value within cluster; voxels are $2 \times 2 \times 2$ mm ${ }^{3}$. The Smoking $>$ Food and

Food $>$ Smoking contrasts were corrected using a voxel threshold of $z=3.09$ (uncorrected $p<0.00 \mathrm{I}$ ), and a minimum cluster size of 70 voxels, whereas the FTND correlation analysis was corrected using a voxel threshold of $z>2.3$ (uncorrected $p<0.01$ ) and a minimum cluster size of 239 voxels.

\section{Correlations with Nicotine Dependence Severity}

Correlations between FTND scores and BOLD response in the Smoking-Baseline contrast resulted in significant positive relationships in a cluster encompassing pregenual/subgenual ACC, dorsal ACC, and right nucleus accumbens, precuneus/posterior cingulate gyrus, right frontal pole, and right insula/superior temporal gyrus (Figure 2a). In addition, a significant negative correlation between FTND and BOLD response was observed in right inferior lateral occipital cortex. In contrast, when examining relationships between FTND scores and BOLD response in the Food - Baseline contrast, we found a significant positive correlation in medial frontal cortex and a negative correlation in right inferior and superior lateral occipital cortex (Figure 2b). Finally, analysis of the relationship between FTND and the Smoking - Food contrast revealed a positive relationship in left insula/temporal pole, left superior/middle temporal gyrus, and dorsal ACC/SMA (Figure 2c and Table 1).

\section{Seed-Based Connectivity}

Right OFC: Results from the PPI analysis indicated increased connectivity with rOFC during Smoking compared with Food videos in bilateral somatosensory cortex, bilateral inferior parietal lobe, and bilateral lateral occipital gyrus/middle temporal gyrus (Table 2 and Figure 3a). In contrast, when examining the increased connectivity with rOFC when watching Food-related videos compared with Smoking videos revealed significant clusters in right frontal pole and posterior cingulate cortex (Figure $3 \mathrm{~b}$ ).

In addition to the main effects, correlations between rOFC connectivity and FTND scores revealed one significant cluster in left middle frontal gyrus, such that connectivity differences with rOFC between smoking cues and food cues was greater for more dependent smokers (Table 2 and Figures $3 b$ and $c)$.

Left insula: Examination of connectivity with left insula revealed increased connectivity during Smoking videos compared with Food videos in right insula (anterior and posterior), left putamen, left OFC/amygdala, bilateral somatosensory cortex, bilateral precuneus, and right occipital pole. When examining those regions that showed greater functional connectivity with insula during Food compared with Smoking videos, we found increased connectivity with right frontal pole (Table 3 and Figures $4 \mathrm{a}$ and $\mathrm{b}$ ).

Examination of the correlations between left insula connectivity differences between smoking and food videos and nicotine dependence severity revealed several significant clusters including left caudate, bilateral thalamus, right insula, right putamen, bilateral pre/post-central gyrus, cuneus, and ACC (dorsal and pregenual) (Table 3 and Figure $4 \mathrm{c})$. 


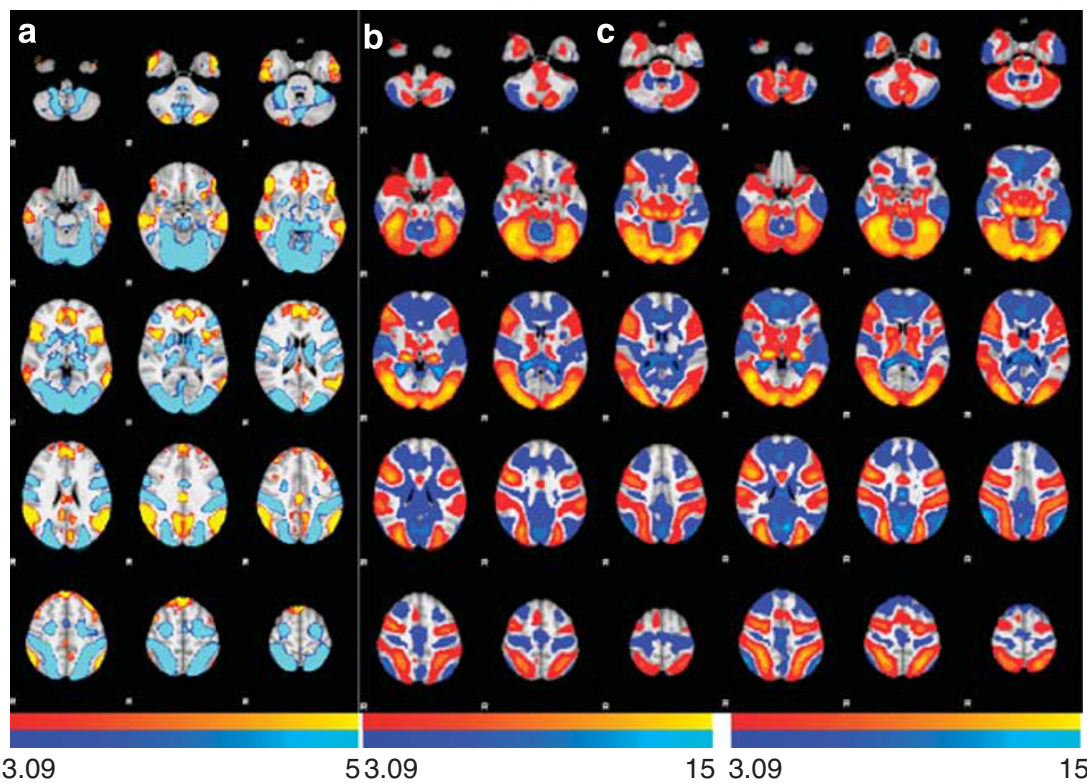

Figure I Blood oxygen level-dependent (BOLD) response during video presentation. (a) Smoking $>$ Food (orange) and Food $>$ Smoking (blue) contrasts $(z>3.09$, corrected $p<0.05$ ). (b) Smoking $>$ Baseline (orange) and Baseline $>$ Smoking (blue). (c) Food $>$ Baseline (orange) and Baseline $>$ Food (blue). ( $b$ and $c$ ) For illustration purposes only, to demonstrate the nature of differences in the Smoking vs Food contrasts. Many of the group differences that emerge are located in regions that show deactivations when compared with baseline. All images are in radiological convention.

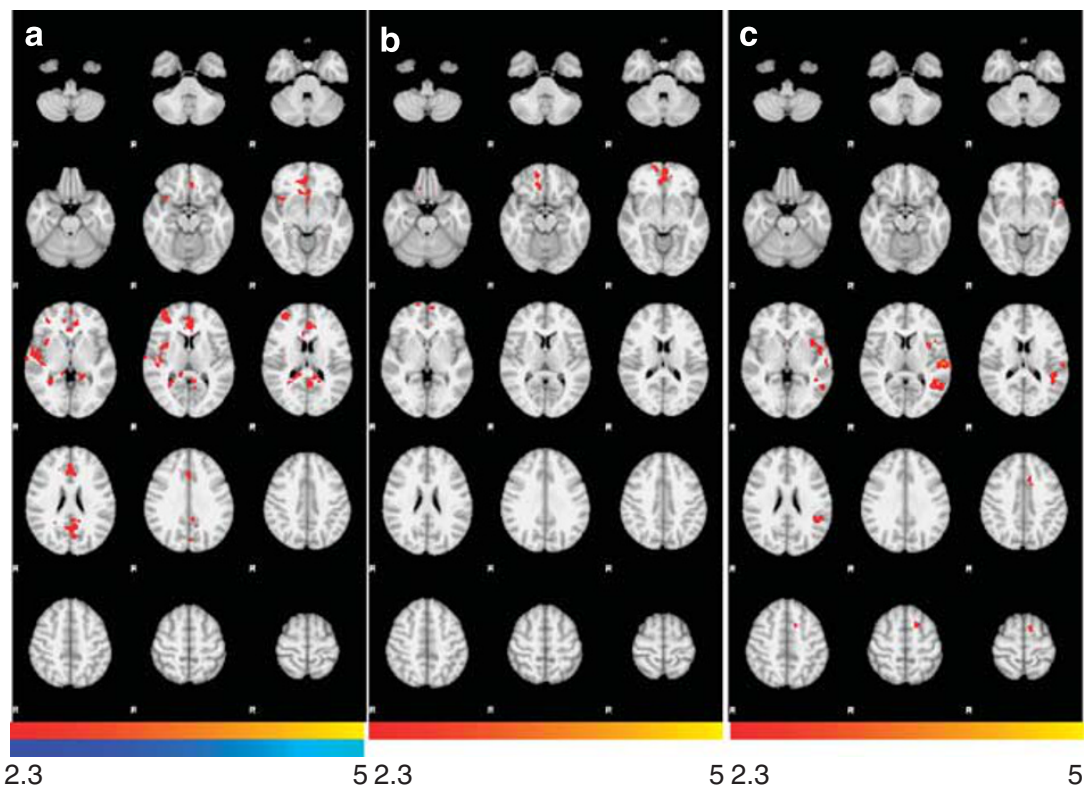

Figure 2 Positive correlation of Fagerstrom test for nicotine dependence (FTND) and blood oxygen level-dependent (BOLD) response. (a) Smoking > Baseline contrast, (b) Food > Baseline, and (c) Smoking > Food. Images are in radiological convention. All maps have been corrected for multiple comparisons.

\section{DISCUSSION}

This study provides compelling evidence of differential response to cigarette cues compared with motivationally significant appetitive cues in smokers in several brain regions, both as a result of enhanced response above baseline and also reduced deactivation below baseline. In addition, functional connectivity analyses showed increased connectivity among networks implicated in motivational valuation and sensorimotor planning, networks that were more strongly connected in smokers with higher levels of nicotine dependence.
Smoking Cue Reactivity Above and Beyond Motivational Cues

Consistent with previous studies that compared neural responses to drug cues and appetitive cues (Claus et al, 2011; Filbey et al, 2008; Wilcox et al, 2011), we found that drug cue-activated regions previously implicated in motivational drive to a greater degree than appetitive food cues. Specifically, smoking videos were associated with greater response in anterior and posterior cingulate gyrus, lateral OFC, dorsal PFC (medial and lateral), MTG, precuneus, and IPL compared with food-related videos. 
Table 2 Regions Showing Differential Connectivity with Right OFC During Smoking and Food Videos

\begin{tabular}{|c|c|c|c|c|c|c|c|}
\hline Contrast & Region & BA & $\operatorname{Max} Z$ & Voxels & $\mathbf{x}$ & $y$ & $\mathbf{z}$ \\
\hline \multirow{5}{*}{ rOFC_Smoking > Food } & Lateral occipital cortex, inferior division (R) & 18 & 4.51 & 1143 & 38 & -82 & 8 \\
\hline & Lateral occipital cortex, superior division (L) & 19 & 4.15 & 888 & -40 & -78 & 18 \\
\hline & Superior parietal lobe $(R)$ & 7 & 3.77 & 850 & 24 & -44 & 68 \\
\hline & Inferior frontal gyrus, pars opercularis (R) & 9 & 4.11 & 491 & 50 & 10 & 22 \\
\hline & Supramarginal gyrus, anterior division $(\mathrm{L})$ & 40 & 3.67 & 484 & -66 & -32 & 34 \\
\hline \multirow[t]{5}{*}{ rOFC_Food > Smoking } & Lateral occipital cortex, superior division (R) & 7 & 3.75 & 443 & 22 & -62 & 58 \\
\hline & Precuneus (R) & 31 & 4.26 & 1626 & 12 & -62 & 32 \\
\hline & Frontal pole (R) & 10 & 4.45 & 1542 & 28 & 58 & -4 \\
\hline & Superior frontal gyrus (L) & 9 & 3.89 & 731 & -22 & 54 & 22 \\
\hline & Inferior temporal gyrus, posterior division $(L)$ & 20 & 3.57 & 557 & -50 & -14 & -34 \\
\hline
\end{tabular}

Coordinates are presented in MNI space. rOFC - Smoking > Food: regions that show enhanced connectivity with right OFC during smoking videos compared with food videos. rOFC - Food > Smoking: regions that show enhanced connectivity with right OFC during food videos compared with smoking videos. rOFC - FTND: regions whose differences in connectivity with rOFC during smoking and food videos positively correlate with FTND scores. BA: Brodmann area; max Z: peak z-value within cluster, voxels are $2 \times 2 \times 2 \mathrm{~mm}^{3}$. All analyses were corrected using a voxel threshold of $z>2.3$ (uncorrected $p<0.0 \mathrm{I}$ ) and a minimum cluster size of 239 voxels.

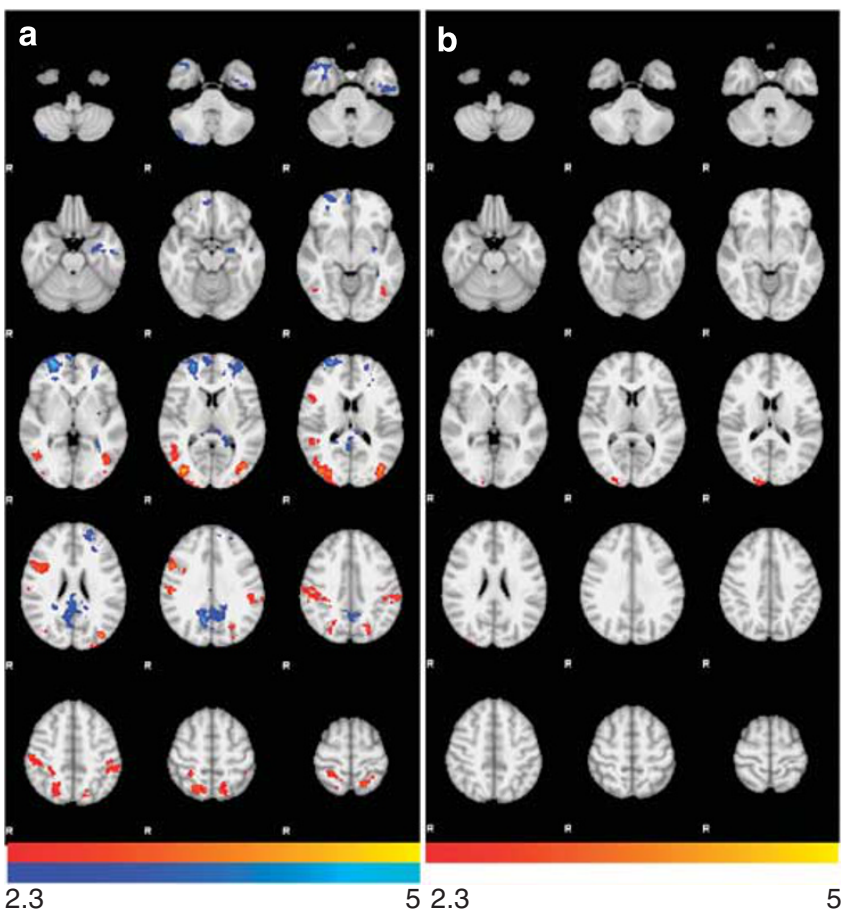

Figure 3 Functional connectivity with right orbitofrontal cortex (OFC). (a) Regions that show increased connectivity with right OFC (rOFC) during smoking videos compared with food videos. (b) Regions that showed negative correlations between Fagerstrom test for nicotine dependence (FTND) and increased connectivity with rOFC during smoking videos compared with food videos. Images are in radiological convention. All maps have been corrected for multiple comparisons.

However, only the bilateral OFC, IPL, and SMA were associated with increases in response above a fixation baseline, whereas the other regions (ACC, PCG, precuneus, and MTG) showed less deactivation below fixation during the smoking videos.

The lateral OFC has been implicated in anticipation of positively and negatively valenced outcomes in decisionmaking studies (Liu et al, 2011, 2007); its presence in this study could be the result of increased craving and/or withdrawal symptoms. The enhanced connectivity between OFC and early visual processing regions suggests that enhanced valuation of smoking cues results in increased allocation of attentional resources, or that early attentional biases develop over continued cigarette use and these visual processing circuits become more integrated with frontal regions involved in valuation. While the directionality of these circuits cannot be determined using connectivity analyses, these results do suggest that enhanced circuit-level neural responses have a role in nicotine dependence.

The IPL and SMA also showed differences between smoking and food cues, with both regions activating above fixation baseline during smoking videos. The IPL has been implicated in attentional bias towards smoking cues (Luijten et al, 2011) and may result in automated responses to cigarettes (Tiffany, 1990, 1999). In concert with the SMA, which is involved in motor planning, enhanced attentional resource allocation towards smoking cues can act to increase the likelihood of smoking and craving (McBride et al, 2006).

A notable finding of this study was the decrease in deactivation within ACC, PCG, MTG, and precuneus during smoking cues than food cues. These regions are considered part of the default-mode network, a system that contributes to self-referential processing and task independent thought (Buckner et al, 2008; Gusnard et al, 2001; Raichle et al, 2001), and typically deactivate during attentionally demanding tasks. The reduced deactivation during the smoking videos suggest that heavier smokers are more engaged in 
Table 3 Regions Showing Differential Connectivity With Left Insula During Smoking and Food Videos

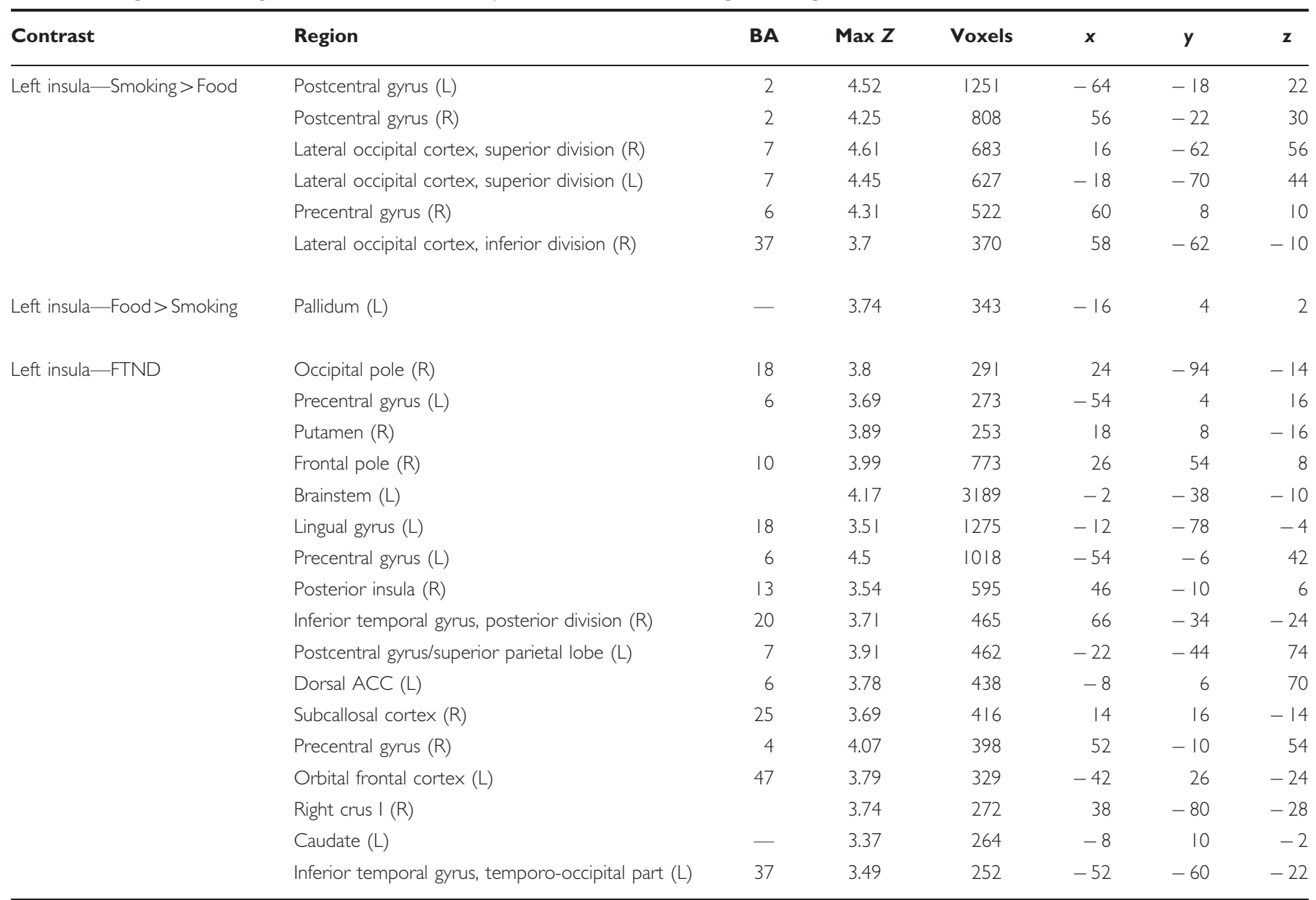

Coordinates are presented in MNI space. Left insula_-Smoking > Food: regions that show enhanced connectivity with left insula during smoking videos compared with food videos. Left insula — Food > Smoking: regions that show enhanced connectivity with left insula during food videos compared with smoking videos. Left insula FTND: regions whose differences in connectivity with left insula during smoking and food videos positively correlate with FTND scores. BA: Brodmann area; max Z: peak $z$-value within cluster; voxels are $2 \times 2 \times 2 \mathrm{~mm}^{3}$. All analyses were corrected using a voxel threshold of $z>2.3$ (uncorrected $p<0.0 \mathrm{I}$ ) and a minimum cluster size of 239 voxels.

self-referential processing such as monitoring craving or withdrawal symptoms. This argument is also supported by the correlations with FTND in the dorsal ACC, with heavier smokers deactivating to a lesser degree than lighter smokers.

\section{Functional Connectivity During Cue Reactivity}

In addition to our novel findings regarding cue reactivity above and beyond motivationally significant cues, PPI was used to identify neural circuits that were more correlated during the presentation of smoking videos than food videos, circuits that may ultimately be responsible for relapse in individuals trying to abstain from smoking. Consistent with the role of OFC in reward and motivational processing, increased connectivity was found with somatosensory cortex and regions involved in early visual processing and object recognition. This network of regions may work by simultaneously increasing attention towards smokingrelated cues and enhancing somatic processing of withdrawal-related cues (Bechara, 2005).
Although connectivity with rOFC appears important to craving responses, the connectivity patterns associated with the insula were more widespread. These results suggest that the anterior insula is part of a network that includes putamen, amygdala, and somatosensory cortex, all of which likely contribute to the initial evaluation of cigarette cue value, interoceptive processing of withdrawal symptoms and/or craving, and the engagement of motoric systems involved in drug-seeking behavior (Bechara, 2005; Koob and Volkow, 2010). Although a previous study found evidence of a functional network that responded during a smoking cue task, confirmation that the network responded preferentially to smoking compared with neutral cues was not provided (Janes et al, 2010). Thus, to our knowledge, this is the first study to report increased connectivity between insula and regions such as amygdala, basal ganglia, and somatosensory cortex during the presentation of smoking cues compared with food cues. This functionally connected network may have a critical role in relapse and thus should be investigated in future studies. 


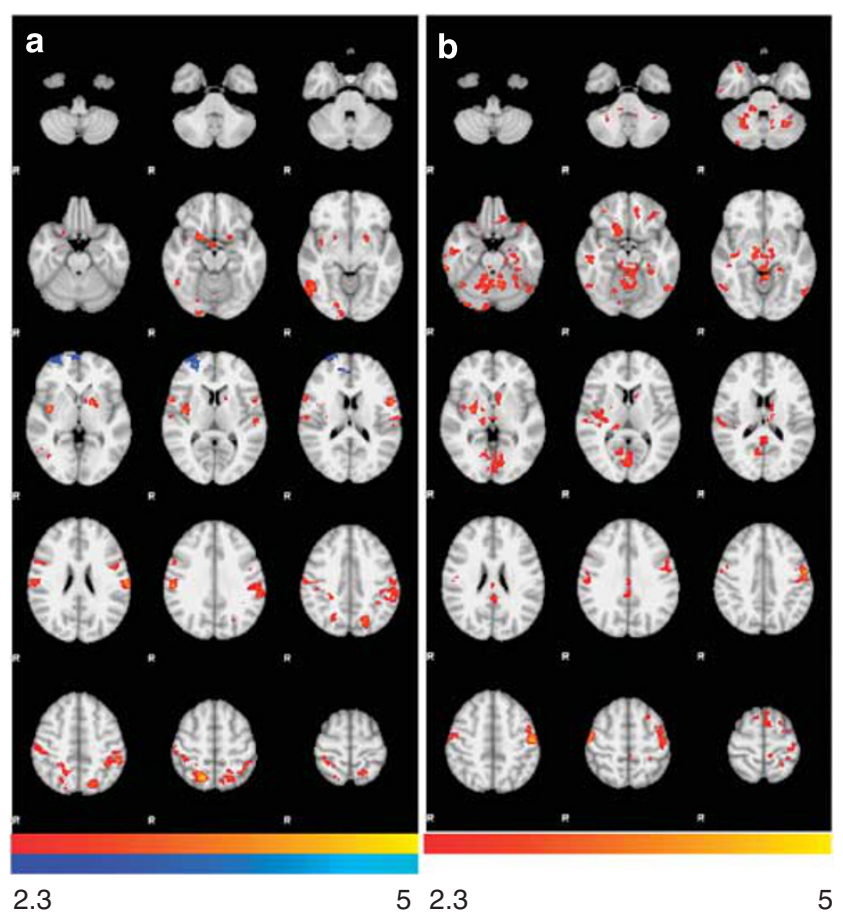

Figure 4 Functional connectivity with left insula. (a) Regions that show increased (orange) and decreased (blue) connectivity with left insula during smoking videos compared with food videos. (b) Regions that showed positive correlations between FTND and greater connectivity with left insula during smoking videos compared with food videos. Images are in radiological convention. All maps have been corrected for multiple comparisons.

\section{Influence of Severity on Neural Responses During Cue Reactivity}

While craving for cigarettes and other drugs of abuse has been well-established as one causal factor in relapse, the mechanisms that underlie craving responses likely change over the course of nicotine dependence as cigarette use is continually reinforced through positive and negative reinforcement. This study attempted to understand the nature of these potential changes by using a large crosssectional sample of smokers with a range of nicotine dependence severity.

Positive correlations between dependence severity and responses during smoking videos were found in subgenual anterior and posterior cingulate, insula, and dorsal ACC, regions that were observed in the overall group comparison. As mentioned above, the fact that anterior and posterior cingulate cortex both are deactivated below baseline, but to a lesser degree in more severely dependent individuals, may suggest that severely dependent smokers engage in more self-referential processing compared with less dependent smokers (Broyd et al, 2009). In contrast, increased response in more severely dependent individuals in insula and dorsal ACC may represent increased interoceptive processing and motor preparation, two processes that may lead to a motivational drive to smoke (Bechara, 2005; Naqvi and Bechara, 2009). FTND scores also showed positive correlations with response differences between smoking and food cues in left insula, temporoparietal junction, and superior frontal gyrus/SMA. Given that the localization of significant correlations with correlations were primarily in posterior cortical regions and not in ventral striatum, these data support the incentive-habit model of addiction (Mogg et al, 2005), wherein greater severity is associated with more reliance on habitual use. While our findings of positive correlations are in direct contrast to the negative correlations reported by (Vollstädt-Klein et al, 2011), it is important to note that there is very little overlap in the regions that showed significant correlations. Further, while our study primarily showed positive correlations in habitlearning circuits, the prior study found negative correlations in more anterior regions implicated in incentive processing.

Finally, when examining correlations between dependence severity and functional connectivity, we found that higher FTND scores were associated with increased connectivity between insula and left caudate, SMA, bilateral pre/post-central gyrus, VTA, subcallosal cortex, right posterior insula/putamen, thalamus, and cuneus. The localization of severity correlations to dorsal striatum, motor/premotor regions, and early visual processing regions further point to a reliance on circuits underlying habitual responding.

\section{Limitations and Future Directions}

While this study has several advantages over previous studies including a large, diverse sample of smokers and a robust motivational control cue, some limitations should be considered. First, this study did not include a control group of non-smokers. While the presence of a control group would identify those regions that differentiate smokers from nonsmokers, previous studies have established group differences including BOLD signal differences in ventral striatum, lateral frontal cortex, intraparietal sulcus, and middle/inferior temporal gyrus (Due et al, 2002; David et al, 2005). Because we were specifically interested in the role of dependence severity on neural engagement, a control group would have been eliminated from severity correlations. Another limitation is a lack of experimental control over the perceptual qualities of the videos used for stimuli. Although we selected videos to include preparation and consumption phases of cigarette use/food, differences observed in regions within the ventral visual processing stream suggest that the cues were not matched in visual properties. In addition, the food videos were not selected based on individual preferences, which could also influence the relative responses throughout the brain. Future studies that utilize personalized food cues that are equivalent in appetitiveness to the cigarette cues would provide a stronger test of craving-specific mechanisms that are modulated by dependence severity.

The results from this study, particularly those from the connectivity analyses, provide a set of a priori regions of interest for future studies of treatment outcomes, as well as providing a better understanding of circuits that could be targeted with pharmaceutical agents. Further, future studies could examine the circuits identified in this study longitudinally to determine the degree to which these circuits change as individuals develop nicotine dependence. Finally, examination of these circuits in smokers who have successfully quit smoking may provide clues about the neural mechanisms that underlie successful behavior change. 


\section{FUNDING AND DISCLOSURE}

The authors declare no conflict of interest.

\section{ACKNOWLEDGEMENTS}

This research was supported by pilot funds from the Mind Research Network and grants from the National Institutes on Alcohol Abuse and Alcoholism (AA012238) and the National Institute on Drug Abuse (DA024002).

\section{REFERENCES}

Bechara A (2005). Decision making, impulse control and loss of willpower to resist drugs: a neurocognitive perspective. Nat Neurosci 8: 1458-1463.

Brody A, Mandelkern M, London E, Childress A, Lee G, Bota R et al (2002). Brain metabolic changes during cigarette craving. Arch Gen Psychiatry 59: 1162-1172.

Broyd SJ, Demanuele C, Debener S, Helps SK, James CJ, SonugaBarke EJS (2009). Default-mode brain dysfunction in mental disorders: a systematic review. Neurosci Biobehav Rev 33: 279-296.

Buckner RL, Andrews-Hanna JR, Schacter DL (2008). The brain's default network: anatomy, function, and relevance to disease. Ann N Y Acad Sci 1124: 1-38.

Bühler M, Vollstädt-Klein S, Kobiella A, Budde H, Reed LJ, Braus DF et al (2010). Nicotine dependence is characterized by disordered reward processing in a network driving motivation. Biol Psychiatry 67: 745-752.

Claus ED, Feldstein Ewing SW, Filbey FM, Sabbineni A, Hutchison KE (2011). Identifying neurobiological phenotypes associated with alcohol use disorder severity. Neuropsychopharmacology 36: 2086-2096.

David SP, Munafò MR, Johansen-Berg H, Smith SM, Rogers RD, Matthews PM et al (2005). Ventral striatum/nucleus accumbens activation to smoking-related pictorial cues in smokers and nonsmokers: a functional magnetic resonance imaging study. Biol Psychiatry 58: 488-494.

Deichmann R, Gottfried J, Hutton C, Turner R (2003). Optimized EPI for fMRI studies of the orbitofrontal cortex. NeuroImage 19: 430-441.

Due D, Huettel S, Hall W, Rubin D (2002). Activation in mesolimbic and visuospatial neural circuits elicited by smoking cues: evidence from functional magnetic resonance imaging. Am J Psychiatry 159: 954-960.

Engelmann JM, Versace F, Robinson JD, Minnix JA, Lam CY, Cui Y et al (2012). Neural substrates of smoking cue reactivity: a metaanalysis of fMRI studies. NeuroImage 60: 1-11.

Filbey FM, Claus E, Audette AR, Niculescu M, Banich MT, Tanabe J et al (2008). Exposure to the taste of alcohol elicits activation of the mesocorticolimbic neurocircuitry. Neuropsychopharmacology 33: 1391-1401.

Franklin T, Wang Z, Wang J, Sciortino N, Harper D, Li Y et al (2007). Limbic activation to cigarette smoking cues independent of nicotine withdrawal: a perfusion fMRI study. Neuropsychopharmacology 32: 2301-2309.

Friston KJ, Buechel C, Fink GR, Morris J, Rolls E, Dolan RJ (1997). Psychophysiological and modulatory interactions in neuroimaging. NeuroImage 6: 218-229.

Gusnard D, Akbudak E, Shulman G, Raichle M (2001). Medial prefrontal cortex and self-referential mental activity: relation to a default mode of brain function. Proc Natl Acad Sci USA 98: $4259-4264$.

Heatherton T, Kozlowski L, Frecker R, Fagerstrom K (1991). The Fagerstrom test for nicotine dependence: a revision of the Fagerstrom tolerance questionnaire. $B r \quad J$ Addict 86: 1119-1127.

Hong LE, Hodgkinson CA, Yang Y, Sampath H, Ross TJ, Buchholz $\mathrm{B}$ et al (2010). A genetically modulated, intrinsic cingulate circuit supports human nicotine addiction. Proc Natl Acad Sci USA 107: 13509-13514.

Janes A, Frederick B, Richardt S, Burbridge C, Merlo-Pich E, Renshaw $\mathrm{P}$ et al (2009). Brain fMRI reactivity to smoking-related images before and during extended smoking abstinence. Exp Clin Psychopharmacol 17: 365-373.

Janes AC, Pizzagalli DA, Richardt S, Frederick B, Chuzi S, Pachas G et al (2010). Brain reactivity to smoking cues prior to smoking cessation predicts ability to maintain tobacco abstinence. Biol Psychiatry 67: 722-729.

Jenkinson M, Bannister P, Brady M, Smith S (2002). Improved optimization for the robust and accurate linear registration and motion correction of brain images. NeuroImage 17: 825-841.

Jezzard P, Matthews P, Smith S (2001). Functional Magnetic Resonance Imaging: An Introduction to Methods. Oxford University Press: New York, NY, USA.

Koob G, Volkow N (2010). Neurocircuitry of addiction. Neuropsychopharmacology 35: 217-238.

Liu X, Hairston J, Schrier M, Fan J (2011). Common and distinct networks underlying reward valence and processing stages: a meta-analysis of functional neuroimaging studies. Neurosci Biobehav Rev 35: 1219-1236.

Liu X, Powell D, Wang H, Gold B, Corbly C, Joseph J (2007). Functional dissociation in frontal and striatal areas for processing of positive and negative reward information. J Neurosci 27: 4587-4597.

Luijten M, Veltman DJ, van den Brink W, Hester R, Field M, Smits $M$ et al (2011). Neurobiological substrate of smoking-related attentional bias. NeuroImage 54: 2374-2381.

McBride D, Barrett S, Kelly J, Aw A, Dagher A (2006). Effects of expectancy and abstinence on the neural response to smoking cues in cigarette smokers: an fMRI study. Neuropsychopharmacology 31: 2728-2738.

McClernon FJ, Kozink RV, Rose JE (2008). Individual differences in nicotine dependence, withdrawal symptoms, and sex predict transient fMRI-BOLD responses to smoking cues. Neuropsychopharmacology 33: 2148-2157.

Mogg K, Field M, Bradley BP (2005). Attentional and approach biases for smoking cues in smokers: an investigation of competing theoretical views of addiction. Psychopharmacology 180: 333-341.

Naqvi N, Rudrauf D, Damasio H, Bechara A (2007). Damage to the insula disrupts addiction to cigarette smoking. Science 315: 531-534.

Naqvi NH, Bechara A (2009). The hidden island of addiction: the insula. Trends Neurosci 32: 56-67.

Oldfield RC (1971). The assessment and analysis of handedness: the Edinburgh inventory. Neuropsychologia 9: 97-113.

O'Reilly JX, Woolrich MW, Behrens TEJ, Smith SM, Johansen-Berg $H$ (2012). Tools of the trade: psychophysiological interactions and functional connectivity. Soc Cogn Affect Neurosci 7: 604-609.

Raichle M, MacLeod A, Snyder A, Powers W, Gusnard D, Shulman G (2001). A default mode of brain function. Proc Natl Acad Sci USA 98: 676-682.

Rubinstein ML, Luks TL, Dryden WY, Rait MA, Simpson GV (2011). Adolescent smokers show decreased brain responses to pleasurable food images compared with nonsmokers. Nicotine Tob Res 13: 751-755.

Smith S, Jenkinson M, Woolrich M, Beckmann C, Behrens T, Johansen-Berg $\mathrm{H}$ et al (2004). Advances in functional and structural MR image analysis and implementation as FSL. NeuroImage 23(Suppl 1): S208-S219.

Smith SM (2002). Fast robust automated brain extraction. Hum Brain Mapp 17: 143-155. 
Smolka MN, Bühler M, Klein S, Zimmermann U, Mann K, Heinz A et al (2006). Severity of nicotine dependence modulates cueinduced brain activity in regions involved in motor preparation and imagery. Psychopharmacology 184: 577-588.

Sobell L, Sobell M (1992). Timeline follow-back: a technique for assessing self-reported alcohol consumption. In Litten RAllen J (eds) Measuring Alcohol Consumption: Psychosocial and Biochemical Methods. Humana Press: New Jersey, USA, pp 41-72.

Tang DW, Fellows LK, Small DM, Dagher A (2012). F ood and drug cues activate similar brain regions: a meta-analysis of functional MRI studies. Physiol Behav 106: 317-324.

Tiffany S (1990). A cognitive model of drug urges and drug-use behavior: role of automatic and nonautomatic processes. Psychol Rev 97: 147-168.

Tiffany S (1999). Cognitive concepts of craving. Alcohol Res Health 23: 215-224.

Vollstädt-Klein S, Kobiella A, Bühler M, Graf C, Fehr C, Mann K et al (2011). Severity of dependence modulates smokers' neuronal cue reactivity and cigarette craving elicited by tobacco advertisement. Addict Biol 16: 166-175.

Welsch SK, Smith SS, Wetter DW, Jorenby DE, Fiore MC, Baker TB (1999). Development and validation of the Wisonsin Smoking Withdrawal Scale. Exp Clin Psychopharmacol 7: 354-361.

Wilcox CE, Teshiba TM, Merideth F, Ling J, Mayer AR (2011). Enhanced cue reactivity and fronto-striatal functional connectivity in cocaine use disorders. Drug Alcohol Depend 115: 137-144.

Wilson S, Sayette M, Delgado M, Fiez J (2005). Instructed smoking expectancy modulates cue-elicited neural activity: a preliminary study. Nicot Tob Res 7: 637-645.

Woolrich M, Behrens T, Beckmann C, Jenkinson M, Smith S (2004). Multilevel linear modeling for fMRI group analysis using Bayesian inference. NeuroImage 21: 1732-1747.

Woolrich M, Ripley B, Brady M, Smith S (2001). Temporal autocorrelation in univariate linear modeling of fMRI data. NeuroImage 14: 1370-1386.

Supplementary Information accompanies the paper on the Neuropsychopharmacology website (http://www.nature.com/npp) 